\title{
Materials synthesis and phase transitions under shock waves
}

\author{
SATISH C GUPTA \\ Condensed Matter Physics Division, Bhabha Atomic Research Centre, Mumbai 400085 , India
}

\begin{abstract}
Shock wave compression of materials is accompanied by high pressure, high-strain-rate loading, elevated temperatures, large shear stresses and excessive plastic deformation. All these features create an unusual state in materials that is not possible in any processing method. Thus, in multi-component system of powders, these can lead to enhanced reactivity because of defect promoted diffusional flow of the reactants in a solid-solid chemical reaction for materials synthesis. On the other hand, in a one component system, these shock compression features may significantly influence the onset pressure, the kinetics, the mechanism and the reversibility of a phase transformation. We. shall illustrate these using examples on the synthesis of $\beta-\mathrm{C}_{3} \mathrm{~N}_{4}$ and our work on the crystal to amorphous phase transitions in $q-\mathrm{GeO}_{2}$ and $\alpha-\mathrm{FePO}_{4}$.
\end{abstract}

Keywords. Shock waves; phase transitions; materials synthesis; superhard materials.

\section{Introduction}

The study of pressure induced phase transitions is important both in basic science and in materials-synthesis applications. A strong correlation between hardness and bulk modulus implies that materials with higher bulk modulus are harder too (Leger et al 1994). For example, diamond, the hardest material known to date, has the highest bulk modulus of $442 \mathrm{GPa}$. Thus, the search for harder materials has led to the identification of materials with higher bulk moduli. Under compression, materials transform to denser phases, which being less compressible, have higher bulk moduli and so higher strength. Diamond and cubic boron nitride, the two hard substances, are the metastable high pressure modifications of carbon and boron nitride, respectively.

Recent calculations have predicted that the $\alpha$ and $\beta$ form of $\mathrm{C}_{3} \mathrm{~N}_{4}$ would have bulk moduli close to that of diamond; moreover, a high pressure cubic polymorph of $\mathrm{C}_{3} \mathrm{~N}_{4}$ may have bulk modulus $10 \%$ more than that of diamond, and be metastable at room pressure (Lue and Cohen 1989, 1990; Teter and Hemley 1996). Experimental measurement of the pressure volume relations by X-ray diffraction technique demonstrated that the bulk modulus of the high pressure phase of transition metal oxide $\mathrm{RuO}_{2}$ is $399 \mathrm{GPa}$, which exceeds $369 \mathrm{GPa}$ of cubic boron nitride and which is only $10 \%$ smaller than that of diamond (Haines et al 1996). Also, the high pressure cubic modification of $\mathrm{MnO}_{2}$ is expected to have bulk modulus exceeding that of diamond (Leger and Haines 1997).

Pressure-induced phase transitions can be caused by both static and shock loading techniques. Under shock waves the nature of compressed state is very different in terms of the duration of compression, rate of loading, non-hydrostatic component of stress, rise in temperature, excessive plastic deformation and production of defects. All these features create an unusual state in materials that is not possible in any processing method. These features could significantly influence the onset pressure, the kinetics, the reversibility, and the mechanism of a phase transition. Under shock loading, the compressed state lasts only for a few microseconds, which makes it extremely difficult to employ microscopic probes like $\mathrm{X}$-ray diffraction and Raman spectroscopy, that are popular with static high pressure experiments. So, a transformation is detected by microscopic measurements. However, all the microscopic techniques can be employed for examining the nature of a phase transition in the samples recovered from various shock pressures; this is possible only for irreversible transitions. Even among irreversible transitions, because of short duration a sluggish transition may not be seen. Generally, kinetics of phase transitions under shock compression is much rapid as compared to that under static pressures. Many phase transitions that take many hours for completion under static loading have been observed to occur within a few microseconds under shock conditions (Duvall and Graham 1977). The causes of this fast kinetics are not well understood.

In case of shock compression of a multicomponent powder mixture, the compressed state generates such an unusual defect state that the chemical reactivity of the constituents is significantly enhanced, and powder mixture can undergo chemical reactions within a few $\mu \mathrm{sec}$ duration shock state (Thadhani 1993). Real-time measurements during shock compression have confirmed the occurrence of chemical reaction in such a short time. In some cases, the chemical reaction does not take place in the shocked state, but the material retrieved after unloading is in highly activated state so that the chemical reactions can occur in the shock modified mixture at 
temperatures substantially lower than and at rates much faster than that in the unshocked materials (Thadhani 1994).

Synthesis of $\beta-\mathrm{C}_{3} \mathrm{~N}_{4}$ has been attempted with shock loading technique (Thadhani 1997). The synthesis of this material has been difficult as. it involves two chemical components, $s p^{3}$ bonded carbon and $s p^{2}$ bonded nitrogen. Earlier, pulse laser ablation and chemical vapour deposition techniques have been used for preparing thin films of $\mathrm{CN}_{x}$ with limited success (Sharma and Narayan 1997). Thadhani (1997) carried out experiments to synthesize $\beta-C_{3} N_{4}$ by shock-induced chemical reaction in precursor containing compound with no $\mathrm{H}$ but excess of $\mathrm{C}$ and $\mathrm{N}$. Sodium dicynamide $\left(\mathrm{NaN}(\mathrm{CN})_{2}\right)$ and covalently bonded tetra cynoethylene $\left(\mathrm{C}_{2}(\mathrm{CN})_{4}\right)$ were selected as cyno group precursors, and blended with sodium azide $\left(\mathrm{NaN}_{3}\right)$ and carbon tetra iodide to provide additional $\mathrm{C}$ and $\mathrm{N}$. Precursors were mixed with $95 \mathrm{wt} \%$ copper powder and packed in steel capsule at $60 \%$ and $85 \%$ of theoretical maximum density. In different experiments samples were recovered after shock loading to $40 \mathrm{GPa}$ and $20 \mathrm{GPa}$ pressure in plate impact experiments employing explosive and gun loading configurations, respectively. The organic residue obtained after removing copper and sodium salt from the shocked sample were examined using infrared spectroscopy (IR) technique. The $40 \mathrm{GPa}$ experiment on tetra cynoethylene showed broad envelop of lines between 900 and $1300 \mathrm{~cm}^{-1}$ (figure 1), which could be assigned to a $\beta-\mathrm{C}_{3} \mathrm{~N}_{4}$ structure. The spectra indicates presence of $\beta-C_{3} N_{4}$ but the structure might have been non-ideal. The IR spectra of samples recorded from gun experiments on sodium dicynamide mixture also showed features corresponding to the formation of $\beta-\mathrm{C}_{3} \mathrm{~N}_{4}$. The experiment with longer shock duration (performed with thicker flying plate) showed greater degree of crystallinity suggesting the influence of kinetic constraints on the crystallization.

For synthesizing a super-hard material, the high pressure phase of a material is required to be retained metastably at room pressure. From this viewpoint it is important to compare phase transitions under static and dynamic loading conditions, as the irreversibility of a phase transition is significantly influenced by the presence of shear and high temperature under shock compression. Shear stresses may cause irreversibility in a transition that is reversible under hydrostatic conditions. The crystal to amorphous phase transition in berlinite form of $\mathrm{AlPO}_{4}$ is reversible or irreversible depending on whether the applied stress is hydrostatic or not (Krugger and Jeanloz 1990; Sankaran et al 1990; Cordier et al 1994a, b; Gillet et al 1995). Our work shows similar behaviour in the crystal to amorphous transition in quartz form of $\mathrm{GeO}_{2}$. On the other hand, increase in temperature during shock compression process may cause reversion to the stable room pressure phase in a transition which otherwise is irreversible under static pressures. This is observed in our work on crystal to amorphous transition in $\alpha-\mathrm{FePO}_{4}$.

In the next two $\S \S$, we present the results of our study on the shock-induced amorphization in $q-\mathrm{GeO}_{2}$ and $\alpha-\mathrm{FePO}_{4}$.

\section{Amorphization of $q-\mathrm{GeO}_{2}$}

The trigonal phase of germanium dioxide $\left(q-\mathrm{GeO}_{2}\right)$ is isomorphic to the $\alpha$-quartz structure of silica, which has been observed to undergo a crystalline to amorphous transition under both static and shock pressures in the 15-35 GPa pressure range (see Sharma and Sikka 1996, and references therein). High resolution electron microscopy measurements on shock recovered samples of quartz revealed two types of disordered materials, one present in the transformation lamellae and the other produced along the microfaults (Gratz et al 1992). The former has been interpreted as arising due to solid-solid transformation (diaplectic glass) and the latter due to quenching of the molten material (fusion glass). Using electron microscopy, even cristobalite (another crystalline phase

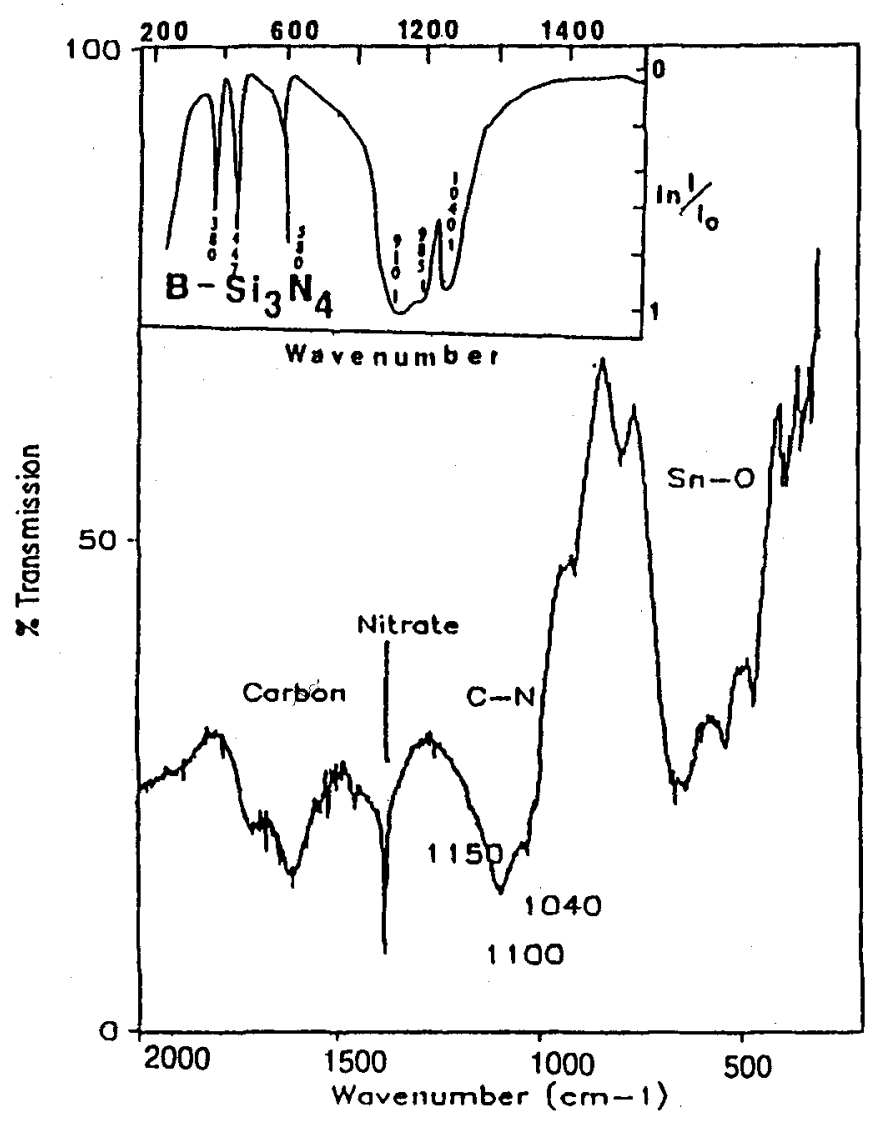

Figure 1. Typical IR spectra of sample showing broad lines between 900 and $1300 \mathrm{~cm}^{-1}$ which could be assigned to $\beta-C_{3} N_{4}$ structure, based on comparison with published IR spectra of $\beta-\mathrm{C}_{3} \mathrm{~N}_{4}$ (Thadhani 1997). 
of $\mathrm{SiO}_{2}$ ) shock amorphized at $\sim 28 \mathrm{GPa}$, has been found to consist of both the diaplectic and fusion glasses (Gratz et al 1993). The objective here was to examine whether the melt quenched effect could be suppressed in an analogous transition that occurs at a lower pressure. In this regard, $q-\mathrm{GeO}_{2}$ is the ideal material to study as it has been reported to amorphize in diamond cell experiments at about $10 \mathrm{GPa}$ (Wolf et al 1992).

The samples of $q-\mathrm{GeO}_{2}$ were shock loaded to the desired pressures by a reverberating shock wave between two stainless steel plates in a recovery capsule upon the impact of flyer plate accelerated in the gas gun (Gupta et al 1995). The retrieved samples were characterized using X-ray diffraction and Raman scattering techniques (Suresh et al 1994). The X-ray diffraction measurements on the shock recovered samples are displayed in figure 2. The pattern of the sample recovered from $5 \mathrm{GPa}$ is identical to the one at ambient conditions. For $6.8 \mathrm{GPa}$, the XRD pattern contains all the original peaks riding on an amorphous background. The peaks are, however, much broadened indicating that lattice strains are present and/or the particle size is reduced. Besides, the pattern also contains two additional peaks ( $d$ spacings $4.04 \AA$ and $3.11 \AA$ ) which do not correspond to any of the known polymorphs of $\mathrm{GeO}_{2}$. The pattern at $7.4 \mathrm{GPa}$ clearly shows that part of the material is amorphized implying that the amorphization has set in between 6.8 and $7.4 \mathrm{GPa}$. The amount of amorphous component increases with pressure, e.g. the pattern for the $10 \mathrm{GPa}$ sample shows a broad hump implying the glassy state of the material. The position of the first glass peak is close to the first peak in the structure factor of the vitreous $\mathrm{GeO}_{2}$ and also to that of the pressure amorphized $\mathrm{GeO}_{2}$ (Leadbetter and Wright 1992) under static conditions. This suggests that the short range order of the amorphous phase obtained in static and dynamic loading might be same. The amorphization of the $q-\mathrm{GeO}_{2}$ was also confirmed by Raman spectra which contained very broad Raman bands for $10 \mathrm{GPa}$ sample. The observation that the amorphization occurs at $7 \mathrm{GPa}$, at much lower pressure of $10 \mathrm{GPa}$ under static pressure supports the view that non-hydrostatic stresses aid the process of amorphization.

The retention of the amorphous phase on shock unloading is in contrast with the complete reversibility of the transition below $10 \mathrm{GPa}$ in hydrostatic environment (Somayazulu et al 1994). The irreversibility of amorphization under shock in $q-\mathrm{GeO}_{2}$ is similar to that in $\mathrm{AlPO}_{4}$, and may be related to the excessive distortion of tetrahedra which do not regain their shapes on unloading.

Electron microscopy measurements on shock recovered $q-\mathrm{GeO}_{2}$ could not be done as the retrieved specimens were pulverized. Therefore, we carried out an estimation of the shear band temperatures in $\mathrm{GeO}_{2}$, in order to assess the possibility of the existence of the fusion glass in the shock recovered samples. A parametric study was performed by varying the strain rate values from $10^{6} \mathrm{~s}^{-1}$ to $10^{8} \mathrm{~s}^{-1}$ and shear band spacings of $2 \mu \mathrm{m}$ and $5 \mu \mathrm{m}$. In figure 3 the plot of the calculated temperatures depicts that the shear band temperatures in $\mathrm{GeO}_{2}$ for all shock pressures upto $10 \mathrm{GPa}$ are below the melting temperature of $1388 \mathrm{~K}$ at ambient (at $10 \mathrm{GPa}$, the melting temperature is even higher). This suggests that the fused $\mathrm{GeO}_{2}$ will

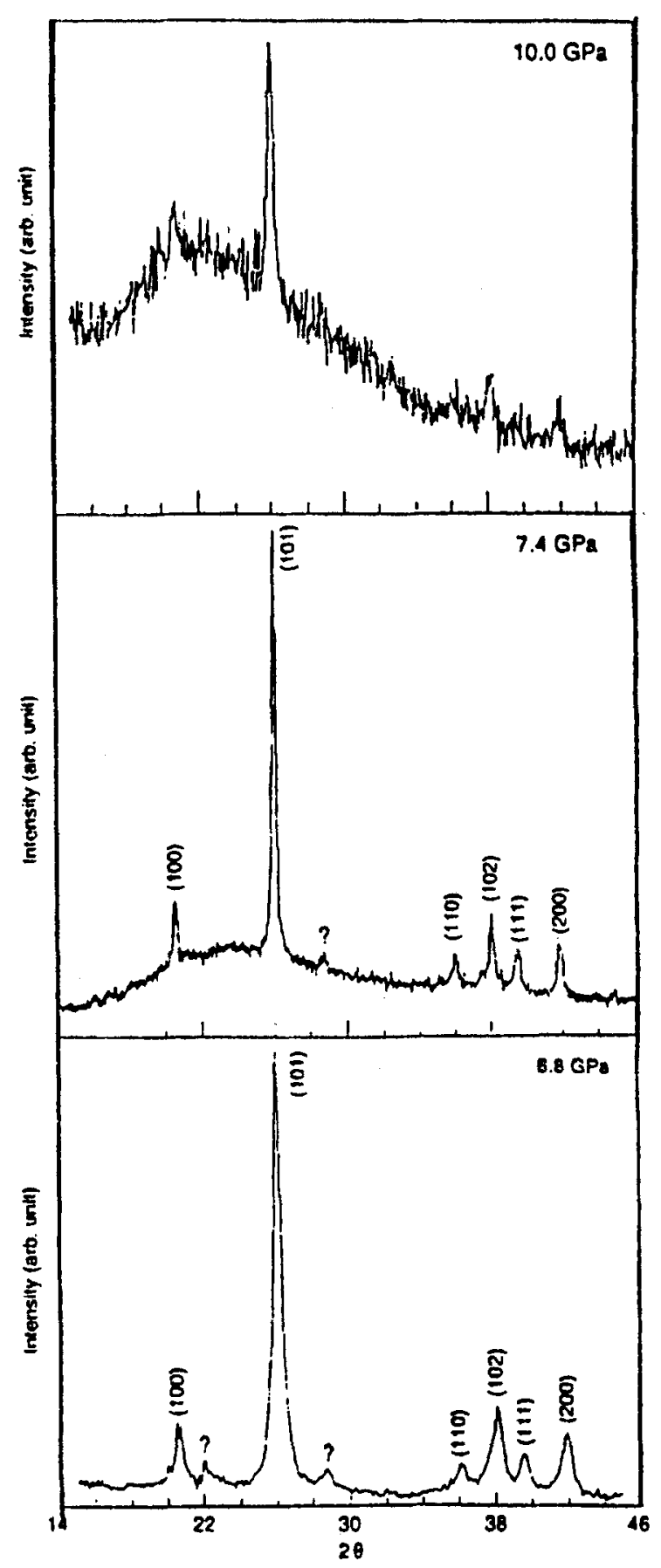

Figure 2. X-ray powder diffraction pattern of $q-\mathrm{GeO}_{2}$ shock recovered from $6.8 \mathrm{GPa}, 7.4 \mathrm{GPa}$ and $10 \mathrm{GPa}$. 
not form up to this shock pressure and implies that the mechanism of shock-induced amorphization in $q-\mathrm{GeO}_{2}$ is a solid-solid one. This inference is supported by the high temperature high pressure work of Yamanaka et al (1992), which reported that the trigonal phase transforms at $85^{\circ} \mathrm{C}$ to rutile form around $0.1 \mathrm{GPa}$ and remains stable at higher pressures, but at lower temperatures the trigonal phase turns amorphous; this amorphous material does not transform to rutile form even on heating to $300^{\circ} \mathrm{C}$. The absence of the rutile phase in the shock recovered specimen confirms that the amorphization occurs in the solid state.

\section{Amorphization in $\alpha$-FePO}

Recently, Chitra et al (1997a, b) conducted static pressure experiments on the berlinite form of iron phosphate $\alpha$-FePO ${ }_{4}$, space group $P 3,21$, in which $\mathrm{Fe}-\mathrm{O}(1)-\mathrm{P}$ and $\mathrm{Fe}-\mathrm{O}(2)-\mathrm{P}$ angles are $139.2^{\circ}$ and $137.5^{\circ}$, respectively. On the basis of Raman measurements in diamond anvil cell, they reported a phase transition around $3 \mathrm{GPa}$, which was confirmed by the XRD measurements that showed the presence of a broad peak which did not disappear till $10 \mathrm{GPa}$. The structure of high pressure phase could not be determined because of the paucity of the diffraction data. We carried out shock wave investigations on $\alpha$ $\mathrm{FePO}_{4}$, in order to identify the structure of this phase and also to compare the behaviour of this material under shock wave and static pressure loading (Joshi et al 1998). Four experiments were conducted on $\alpha$-FePO with initial density of $1.8 \mathrm{~g} / \mathrm{cm}^{3}$ (porosity $40 \%$ ), a nominal diameter of $13 \mathrm{~mm}$ and $0.9 \mathrm{~mm}$ thickness. The samples were emplaced in a recovery fixture (Gupta 1996) and loaded to shock pressure up to $8.5 \mathrm{GPa}$ using the gas gun.

The pressure history in the sample was estimated by performing numerical simulations using a two-dimen-

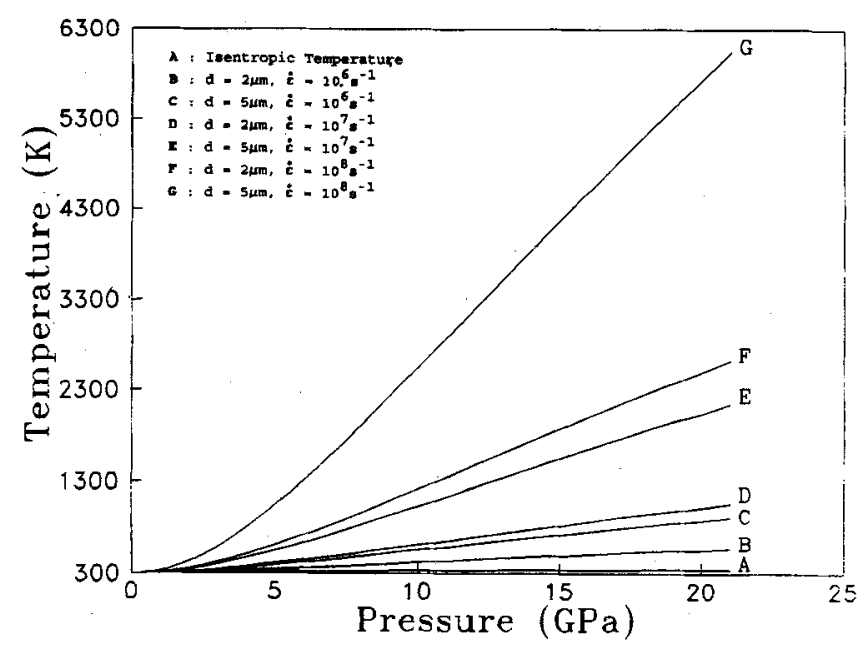

Figure 3. Calculated shear band and isentropic temperatures as a function of shock pressure in $\mathrm{GeO}_{2}$. sional hydrodynamic code with the measured projectile velocity and estimated Hugoniot of $\mathrm{FePO}_{4}$ as input. The peak pressures for the four experiments corresponding to the measured projectile velocities of $0.11,0.19,0.27$ and $0.45 \mathrm{~km} / \mathrm{sec}$ were estimated to be $2(0.5), 3.5(0.5)$, $5 \cdot 2(0.5)$ and $8 \cdot 5(0.5) \mathrm{GPa}$, respectively. A typical calculated pressure profile for sample shock loaded to $8.5 \mathrm{GPa}$ is shown in figure 4. As shown in the figure, sample reached the final pressure after a few reverberations and its peak pressure lasted for around 1-2 $\mu$ s. The shock recovered samples were characterized using powder X-ray diffraction.

The XRD measurements on the shock recovered samples are displayed in figure 5. The XRD pattern of the sample recovered from $2 \mathrm{GPa}$ is identical to that at ambient conditions. However, for $3.5 \mathrm{GPa}$ sample, the diffraction pattern exhibits new peaks along with the broadening of the peaks of the original phase. The development of these peaks is more clear in XRD pattern of the sample pressurized up to $5.2 \mathrm{GPa}$. These new diffraction peaks could be associated with an orthorhombic phase having space group $\mathrm{Cmcm}$ and cell constants $a=5.227 \AA, b=7.770 \AA, c=6.322 \AA$ (Kinomura 1976). Moreover, the peaks are riding over hump like background indicating the presence of amorphous material. This suggests that the trigonal phase has irreversibly transformed partly to orthorhombic crystalline phase and partly to the amorphous phase around $5 \mathrm{GPa}$. The coexistence of the high pressure crystalline phase and the amorphous phase is quite unusual.

It is interesting to compare the above results with those of the static pressure measurements of Chitra et al (1997a) performed concurrently in our laboratory. In the in situ XRD measurements on the sample above $3 \mathrm{GPa}$, Chitra et al (1997a) observed the disappearance of the strong (012) original peak (of the trigonal structure) with

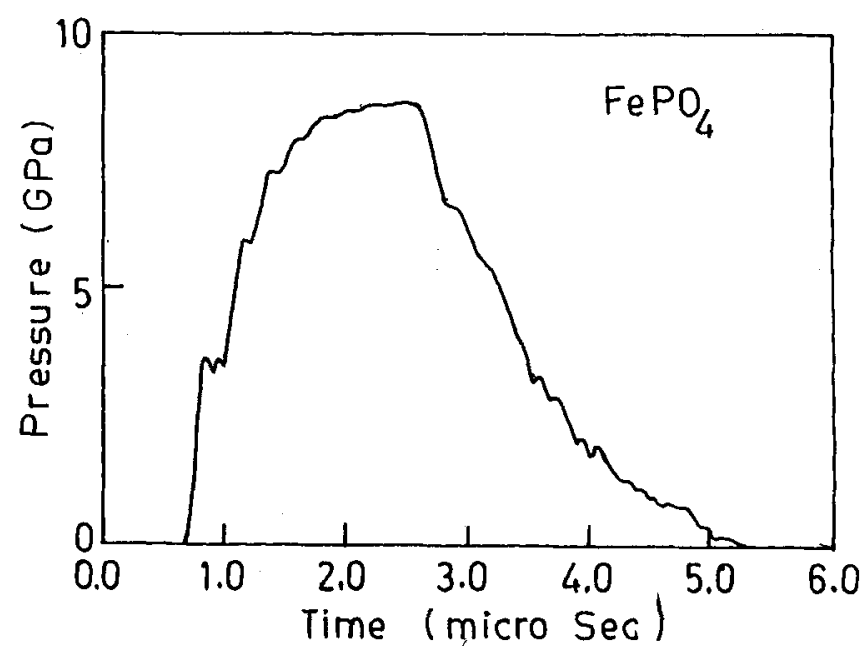

Figure 4. Pressure history in the $\mathrm{FePO}_{4}$ sample calculated using a two-dimensional hydrodynamic code. The projectile velocity in this case is $0.448 \mathrm{~km} / \mathrm{s}$. 
emergence of a weaker peak close to it and appearance of a new broad peak near (110) original peak. This indicated a transition to new crystalline phase. However, because of paucity of the diffraction data, the new structure could not be determined. On comparing our XRD pattern with that of Chitra et al (1997a) we found that the new unidentified peaks seen under static pressure belonged to the orthorhombic structure observed in the shocked samples, and suggested that the unidentified high pressure structure reported by Chitra et al could be orthorhombic. This was confirmed by the later XRD measurements (Chitra et al 1997b) on the pressure quenched samples prepared by compressing above $3 \mathrm{GPa}$ between tungsten carbide anvils in a 100 ton press.

The XRD pattern of the sample shock recovered from 8.5 GPa shows that in comparison with that of the 5.2 GPa pattern the amorphous background has decreased, the peaks belonging to the orthorhombic structure are much weaker and the peaks corresponding to the parent trigonal phase are sharper. Two experiments gave almost similar results. This behaviour of $\alpha-\mathrm{FePO}_{4}$ is abnormal and is in contrast to that of $q-\mathrm{GeO}_{2}$ (Suresh et al 1994) where the proportion of the amorphous component in the shock retrieved samples increased with the peak loading stress.

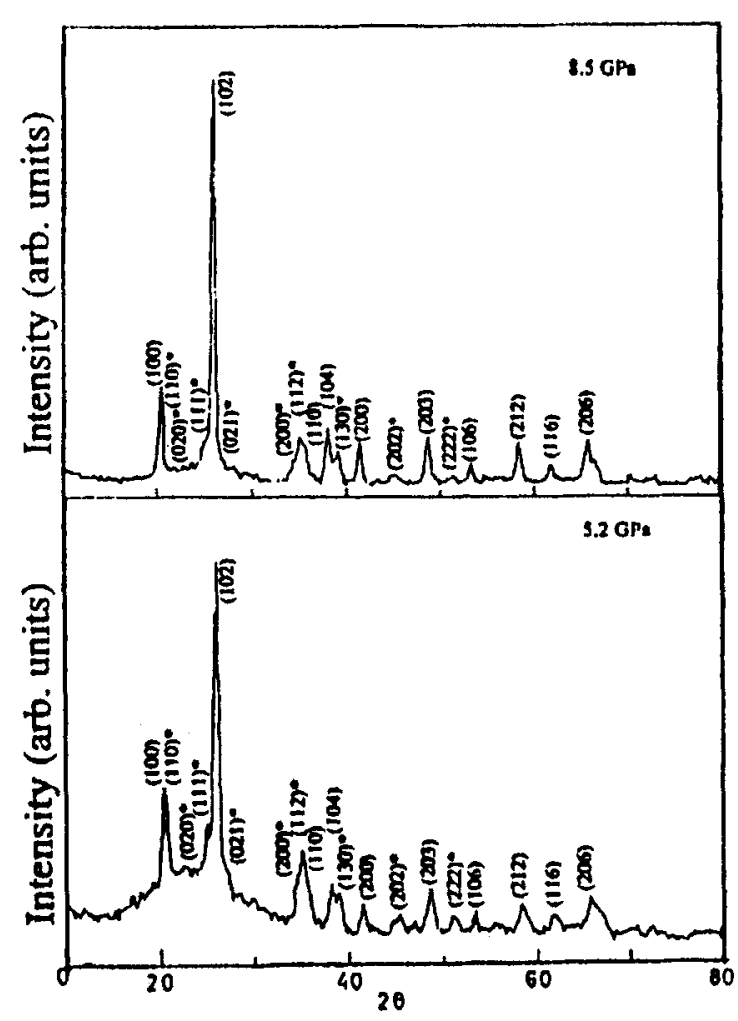

Figure 5. X-ray diffraction pattern of $\mathrm{FePO}_{4}$ sample recovered from $5.2 \mathrm{GPa}$ and $8.5 \mathrm{GPa}$. The reflections $(h k l)$ belong to the trigonal structure whereas $(h k l)^{*}$ correspond to the orthorhombic structure.
It is heartening to note that the later high pressure experiments on $\alpha-\mathrm{FePO}_{4}$ (Pasternak et al 1997) done at CHESS Synchrotron source found the transition to coexisting crystalline orthorhombic structure and amorphous phase, in agreement with our observations.

As the $\mathrm{Cmcm}$ phase is the equilibrium phase under pressure for this family of phosphate compound (Sharma and Sikka 1995), the above results could be interpreted in terms of a three-level free energy diagram. Figure 6 shows a schematic diagram of the free energy versus the reaction coordinate, where $G_{c 1}, G_{a}$ and $G_{c 2}$ are the free energies of the initial crystalline phase $c_{1}$ just before the transformation, amorphous phase $a$, and the high pressure phase $c_{2}$, respectively; $\Delta g_{a}, \Delta g_{\mathrm{c} 2}$ and $\Delta g_{c 1 \mathrm{c} 2}$ (not shown in figure) are the barrier heights for $c_{1}$ to $a, a$ to $c_{2}$ and $c_{1}$ to $c_{2}$ transitions, respectively. The compressed berlinite phase $\left(c_{1}\right)$ energetically prefers to transform to the $\mathrm{Cmcm}$ phase $\left(c_{2}\right)$ above $3.5 \mathrm{GPa}$. However, the kinetics impedes this and instead a transformation to the amorphous (a) phase occurs. This then transforms to the equilibrium phase. This is similar to the behaviour of quartz $\mathrm{SiO}_{2}$, where the $c \rightarrow a$ transition occurs at $21 \mathrm{GPa}$ and the traces of the equilibrium stishovite phase are detected at $70 \mathrm{GPa}$ (Hazen and Finger 1979, 1984, 1985; Moffat et al 1980). The fact that both the transitions in $\mathrm{FePO}_{4}$ occur in a small pressure interval suggests that the energy barriers $\Delta g_{a}$ and $\Delta g_{c 2}$ are of similar magnitude. However, we can not rule out the direct $c_{1}$ to $c_{2}$ transformation which depends on the barrier $\Delta g_{\text {ckc2 }}$. Now, in the case of $8.5 \mathrm{GPa}$ experiment, although higher component of both the amorphous phase and orthorhombic phase would have formed, these might have not been retained on unloading due to the reverse transformation caused by the higher residual temperature as our sample has lot of porosity. This leads to the presence of very small components of the amorphous as well as higher pressure structure.
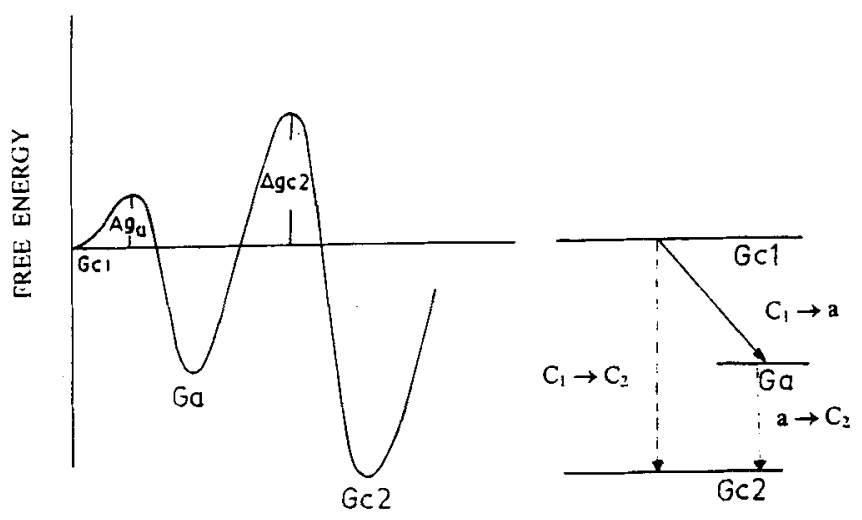

REACTION COORDINATE

Figure 6. A schematic diagram showing free energy as a function of reaction coordinate and the three-level free energy diagram for crystal to amorphous transition. 
We find the onset of shock induced amorphization in $\alpha$-FePO ${ }_{4}$ around $3 \mathrm{GPa}$ along with transition to an orthorhombic phase. However, unlike under static high pressures where the amount of amorphous material continues to increase on increasing the pressure, under shock compression proportion of the amorphous material it has substantially decreased at $8.5 \mathrm{GPa}$, because of the reversion due to higher post shock temperature. Our results support the validity of the three-level free energy diagram for interpreting $c \rightarrow a$ transitions.

\section{Acknowledgements}

I thank Dr S K Sikka for useful discussions.

\section{References}

Chitra M, Sharma S M, Kulshreshtha S K and Sikka S K 1997a Pramana-J. Phys. 49285

Chitra V, Momin S N, Kulshreshtha S K, Sharma S M and Sikka S K 1997b in Advances in high pressure research in condensed matter (eds) Sikka S K, Gupta S C and Godwal B K (New Delhi: NISCOM) pp. 331-336

Cordier P, Doukhan J C and Peyronneau J 1994a Phys. Chem. Miner. 20176

Cordier P, Gratz A J, Doukhan J C and Nellis W J 1994b Phys. Chem. Miner. 21133

Duvall G E and Graham R A 1977 Rev. Mod. Phys. 49523

Gillet P, Badro J, Varrel B and Macmillan P F 1995 Phys. Rev. B51 11262

Gratz A J, Nellis W J, Christie J M, Brocious W, Swegle J and Cordier P 1992 Phys. Chem. Miner. 19267

Gratz A J, DeLoach L D, Clough T M and Nellis W J 1993 Science 259663

Gupta S C 1996 Indian J. Pure \& Appl. Phys. 34651

Gupta S C, Agarwal R G, Gyanchandani J S, Roy S, Suresh N, Sikka S K, Kakodkar A and Chidambaram R 1992 Shock compression of condensed matter-1991 (eds) S C Schmidt, R D Dick, J W Forbes and D G Tasker (Amsterdam: Elsevier) pp. 839

Gupta S C, Jyoti G, Suresh N, Sikka S K, Chidambaram R, Agarwal R G, Roy S and Kakodkar A 1995 Bhabha Atomic Research Centre Report Number BARC/1995/E/017
Haines J, Leger J M and Schutte O 1996 Science 271629

Hazen R M and Finger L W 1979 Phase Trans. 11

Hazen R M and Finger L W $1984 \mathrm{Am}$. Sci. 72143

Hazen R M and Finger L W 1985 Sci. Am. 252110

Joshi K D, Suresh N, Jyoti G, Kulshreshtha S K, Gupta S C and Sikka S K 1998 Shock Waves 8173

Kinomura N 1976 Mater. Res. Bull. 11457

Krugger M B and Jeanloz R 1990 Science 249647

Leadbetter A J and Wright A C 1992 Non-Crystalline Solids 7 37

Leger J M, Haines J and Blanzat B 1994 J. Mater. Sci. Lett. 131688

Leger J M and Haines J 1997 Endeavour 21121

Lue A Y and Cohen M L 1989 Science 245841

Lue A Y and Cohen M L 1990 Phys. Rev. B41 10722

Moffat W G, Pearsall G W and Wulff J 1980 Structure and Properties of Materials (New Delhi: Wiley Eastern Ltd.) vol. 1 Ch. 5

Pastemak M P et al 1997 Phys. Rev. Lett. 794409

Sankaran H, Sharma S M, Sikka S K and Chidambaram R 1990 Pramana-J. Phys. 35177

Sharma S M and Sikka S K 1995 Phys. Rev. Lett. 74 3301

Sharma S M and Sikka S K 1996 Prog. Mater. Sci. 401 (and references therein)

Sharma A K and Narayan J 1997 Int. Mater, Rev. 42137

Somayazulu M S, Garg N, Sharma S M and Sikka S K 1994 Pramana-J. Phys. 431

Suresh N, Jyoti G, Gupta S C, Sikka S K, Sangeeta and Sabharwal S C $1994 \mathrm{~J}$. Appl. Phys. 761530

Teter D M and Hemley M J 1996 Science 27153

Thadhani N N 1993 Prog. Mater. Sci. 37117

Thadhani N N 1994 J. Appl. Phys. 762129

Thadhani N N 1997 in Advances in high pressure research in condensed matter (eds) S K Sikka, Gupta S C and B K Godwal (New Delhi: NISCOM) pp. 214-221

Wolf G H, Wang S, Herbert C A, Durben D J, Oliver W F, Kang Z C and Halvorson K 1992 High pressure physics: application to earth and planetary science (eds) $\mathrm{Y}$ Syono and M H Manghnani (Tokyo: Terra Scientific) and (Washington DC: American Geophysical Union) p. 503

Yamanaka T, Shibata T, Kawasaki S and Kume S 1992 in High pressure research: Application to earth and planetary sciences (eds) Y Syono and M H Manghnani 493-501 